Article

\title{
Lipid Stores and Lipid Metabolism Associated Gene Expression in Porcine and Bovine Parthenogenetic Embryos Revealed by Fluorescent Staining and RNA-seq
}

\author{
Arkadiusz Kajdasz ${ }^{1}$ (D) Ewelina Warzych ${ }^{2}$, Natalia Derebecka ${ }^{3}$, Zofia E. Madeja ${ }^{2}$, \\ Dorota Lechniak ${ }^{2}$, Joanna Wesoly ${ }^{3}$ and Piotr Pawlak ${ }^{2, *} \mathbb{D}$ \\ 1 Laboratory of Human Molecular Genetics, Institute of Molecular Biology and Biotechnology, \\ Faculty of Biology, Adam Mickiewicz University, 61-614 Poznan, Poland; akajdasz@amu.edu.pl \\ 2 Department of Genetics and Animal Breeding, Faculty of Veterinary Medicine and Animal Sciences, \\ Poznan University of Life Sciences, Wolynska 33, 60-637 Poznan, Poland; \\ ewelina.warzych@up.poznan.pl (E.W.); zofia.madeja@up.poznan.pl (Z.E.M.); \\ dorota.cieslak@up.poznan.pl (D.L.) \\ 3 Laboratory of High Throughput Technologies, Institute of Molecular Biology and Biotechnology, Faculty of \\ Biology, Adam Mickiewicz University, Umultowska 89, 61-614 Poznan, Poland; nataliad@amu.edu.pl (N.D.); \\ j.wesoly@amu.edu.pl (J.W.) \\ * Correspondence: piotr.pawlak@up.poznan.pl; Tel.: +48-61846-6111
}

Received: 21 July 2020; Accepted: 3 September 2020; Published: 5 September 2020

check for updates

\begin{abstract}
Compared to other mammalian species, porcine oocytes and embryos are characterized by large amounts of lipids stored mainly in the form of droplets in the cytoplasm. The amount and the morphology of lipid droplets (LD) change throughout the preimplantation development, however, relatively little is known about expression of genes involved in lipid metabolism of early embryos. We compared porcine and bovine blastocyst stage embryos as well as dissected inner cell mass (ICM) and trophoblast (TE) cell populations with regard to lipid droplet storage and expression of genes functionally annotated to selected lipid gene ontology terms using RNA-seq. Comparing the number and the volume occupied by LD between bovine and porcine blastocysts, we have found significant differences both at the level of single embryo and a single blastomere. Aside from different lipid content, we found that embryos regulate the lipid metabolism differentially at the gene expression level. Out of 125 genes, we found 73 to be differentially expressed between entire porcine and bovine blastocyst, and 36 and 51 to be divergent between ICM and TE cell lines. We noticed significant involvement of cholesterol and ganglioside metabolism in preimplantation embryos, as well as a possible shift towards glucose, rather than pyruvate dependence in bovine embryos. A number of genes like DGAT1, CD36 or NR1H3 may serve as lipid associated markers indicating distinct regulatory mechanisms, while upregulated PLIN2, APOA1, SOAT1 indicate significant function during blastocyst formation and cell differentiation in both models.
\end{abstract}

Keywords: blastocyst; lipid droplet; parthenogenesis; lipid metabolism; gene expression; RNA-seq; pig; cattle

\section{Introduction}

The patterns according to which the genes are expressed provide biological insight into their function. Tissue and species specificity, imprinting, methylation, temporal and allele specificity are just a few regulatory mechanisms describing regulation of gene expression in developmental biology. 
For over two decades, microarrays have been used to investigate expression of thousands of genes in different species depending on the development of technology and reference genomes. Over time, microarrays have been replaced by next generation sequencing platforms that enable sequencing of genomes on an unprecedented scale. In embryology, the breakthrough came with the work of Tang et al., who, for the first time, described global gene expression in mouse embryos at a single cell level by RNA-seq [1]. Moreover, the expression profile of individual blastomeres of human preimplantation embryos, as well as embryonic stem cell lines, were published in 2013 [2]. Since then, the RNA-seq technology revolutionized the embryology of model and laboratory animals as well as of livestock science. Nowadays pigs and cattle are considered to be the best animal models for biomedical applications. This is acknowledged due to the similarities in physiology and pathology on one hand, and the kinetics of preimplantation embryo development, timing of genome activation and blastocyst formation on the other [3].

Compared to other livestock animals, porcine oocytes and embryos are characterized by high lipid content stored mainly in the form of droplets in the cytoplasm [4-8]. Lipid droplets (LD) are dynamic organelles with important roles in lipid metabolism. LD consist largely of neutral lipids, predominantly triglyceride (TG) and cholesterol ester (CE) which provide substrates for energy production, membrane components and signaling lipids $[9,10]$. LD are thought to be formed de novo in the endoplasmic reticulum. Mature LD consist of a neutral lipid core coated with a phospholipid monolayer and structural proteins such as perilipins, regulators of LD function [11,12]. During mammalian oogenesis, LD are stored in the ooplasm, however, for unknown reasons, the exact content of LD varies widely among species $[6,13]$. Moreover, LD morphology changes dynamically during early embryonic development and in response to culture conditions [14,15].

Histochemical analysis of porcine embryos showed changes in lipid composition during preimplantation development, along with the observed differences between in vivo and in vitro derived embryos [16]. The LD content remained constant up to the morula stage and it decreased substantially during the blastocyst formation. With regard to bovine embryos, lipid content was observed to be constant up to the 8/16-cell stage, and was followed by a significant increase at the morula stage and a decrease in blastocysts [17]. Therefore, in both species, embryos at the blastocyst stage probably intensify $\beta$-oxidation to support increased energy demand during blastocyst formation, blastocoel expansion and hatching. It is supported by data summarized by Sturmey et al., who showed that in mice, cows and pigs, oxygen consumption greatly increases at the blastocyst stage [18]. In comparison to other species, the highest values of oxygen consumption were observed in pig embryos at all stages of preimplantation development. However, still relatively little is known about genes encoding factors involved in lipid metabolism during the preimplantation development. Significant amounts of lipids and hypothetical scenarios of lipid utilization at the time of implantation indicate their important function for early development. Therefore, unravelling the metabolic pathways and the genes involved in the processes of lipogenesis/lipolysis is crucial to understand the biology of preimplantation embryo as well as to optimize embryo culture conditions in vitro.

\section{Results}

\subsection{Embryo Culture}

Altogether, 186 and 214 porcine and bovine oocytes respectively were in vitro matured and activated for parthenogenetic development. A total of 89\% (165/186) and 91\% (197/214) of activated porcine and bovine oocytes cleaved within $24 \mathrm{~h}$ of culture. Blastocyst yield accounted for $36 \%$ for porcine ( 7 days postactivation $-7 \mathrm{dpa}$ ) and $21 \%$ for bovine embryos ( $8 \mathrm{dpa}$ ). Mean number of blastomeres accounted for $30(+/-11)$ in porcine and $63(+/-17)$ in bovine embryos. 


\subsection{Lipid Droplet Characteristics}

Altogether, 32 parthenogenetic blastocysts (18 porcine and 14 bovine) were analyzed for lipid storage (LD number and volume; Figure 1). Porcine embryos were abundantly packed with LD. Quantitative analysis revealed a positive correlation of lipid droplet number (per blastomere) with their volume only in bovine embryos (Figure $2 p<0.01 r=0.84$ ). No correlation was found for porcine embryos and blastomeres which may result from heterogeneous population of LD differing in size. With regard to the entire embryo, a similar number of LD was present in bovine and porcine blastocysts, however, their volume was lower in bovine compared to pig embryos, due to morphological LD divergence between species (Figures 1 and $2 p<0.05$ ). We observed significantly more LD per single blastomere in porcine embryos, a difference that arises from significantly higher number of cells in bovine embryos. According to our previous observations that LD number does not fully describe the lipid storage, we analyzed the volume occupied by LD in embryos and blastomeres. We noticed that LD exhibited significantly higher volume in porcine entire embryos and single blastomeres (Figure 2 $p<0.05)$.

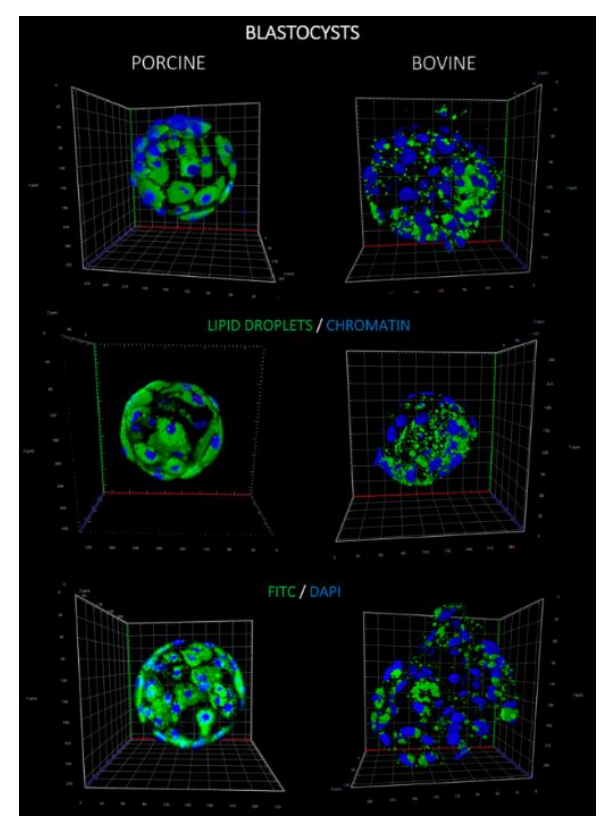

Figure 1. Confocal 3D projections of fluorescently stained porcine (left) and bovine (right) blastocyst stage embryos with BODIPY 493/503 (green) to visualize lipid droplets and DAPI (4',6-diamidino2-phenylindole; blue) for nucleus staining.

\subsection{RNA Sequencing of Porcine and Bovine Blastocyst Embryos}

Parthenogenetic embryos were collected at day 7 pa and day 8 pa of development for pig and bovine, respectively. Individual embryos as well as dissected inner cell mass (ICM) and trophoblast (TE) cell populations were sequenced. Altogether 18 samples have been analyzed ( 8 bovine and 10 porcine). The RNA-seq dataset has been deposited in GEO: accession GSE156149. The number of raw reads (pair-end) ranged from 22 to 65 million per sample for porcine embryos and from 34 to 53 million per sample for bovine. On average 11515 features (genes) were found after orthologues selection by NOIseq across the samples (Figure S1, Table S1). According to Gene ontology (GO) "lipid_droplet" term we found 44 out of 63 differentially expressed genes (DEG; $p<0.05$; Figure 3) between whole porcine and bovine blastocyst; 19 between porcine and bovine ICMs and 23 between porcine and bovine TEs. Moreover, interspecies comparisons of ICM and TE populations showed 6 and 1 DEGs in bovine and porcine cell lines, respectively. A total of 17 genes were upregulated in porcine embryos, while bovine showed 27 genes significantly upregulated. With respect to blastocyst two cell lines we noticed 14 vs. 7 (ICM) and 10 vs. 12 (TE) significantly upregulated genes in porcine 
and bovine embryos, respectively. Gene ontology "lipid_storage" term with known 62 genes showed 34 DEGs between bovine and porcine blastocysts $(p<0.05$; Figure 4$)$. A total of 18 genes in whole porcine embryos and 14 genes in bovine were significantly upregulated, whereas ICM showed 19 and TE 23 DEGs. Among them, 13 vs. 6 (ICM) and 16 vs. 7 (TE) were significantly upregulated in porcine and bovine embryos, respectively.
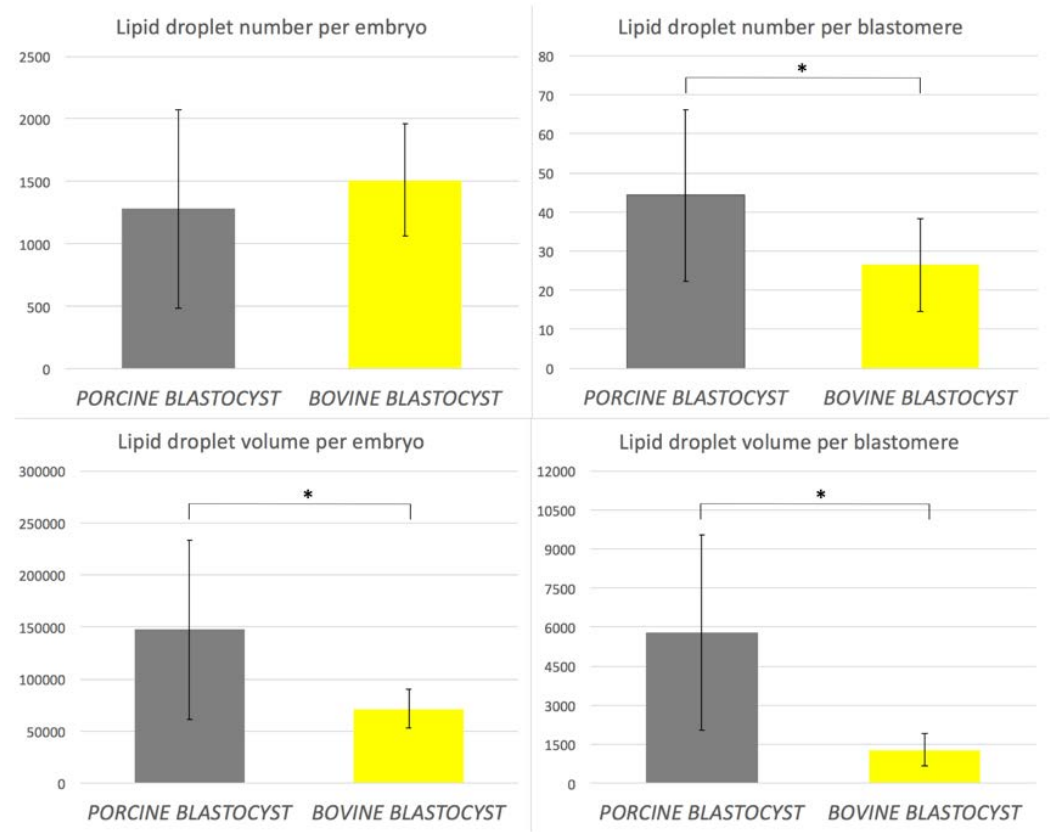

Figure 2. Lipid droplet number and volume calculated per single embryo and per blastomere in blastocyst stage embryos using Fiji software and 3D images captured with confocal microscope. Data are presented as mean $+/-\mathrm{SD}$. Significant differences are marked with asterisks $\left({ }^{*} p<0.05\right)$.

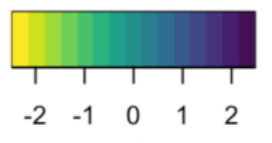

\section{Lipid droplet GO term}

Column Z-Score

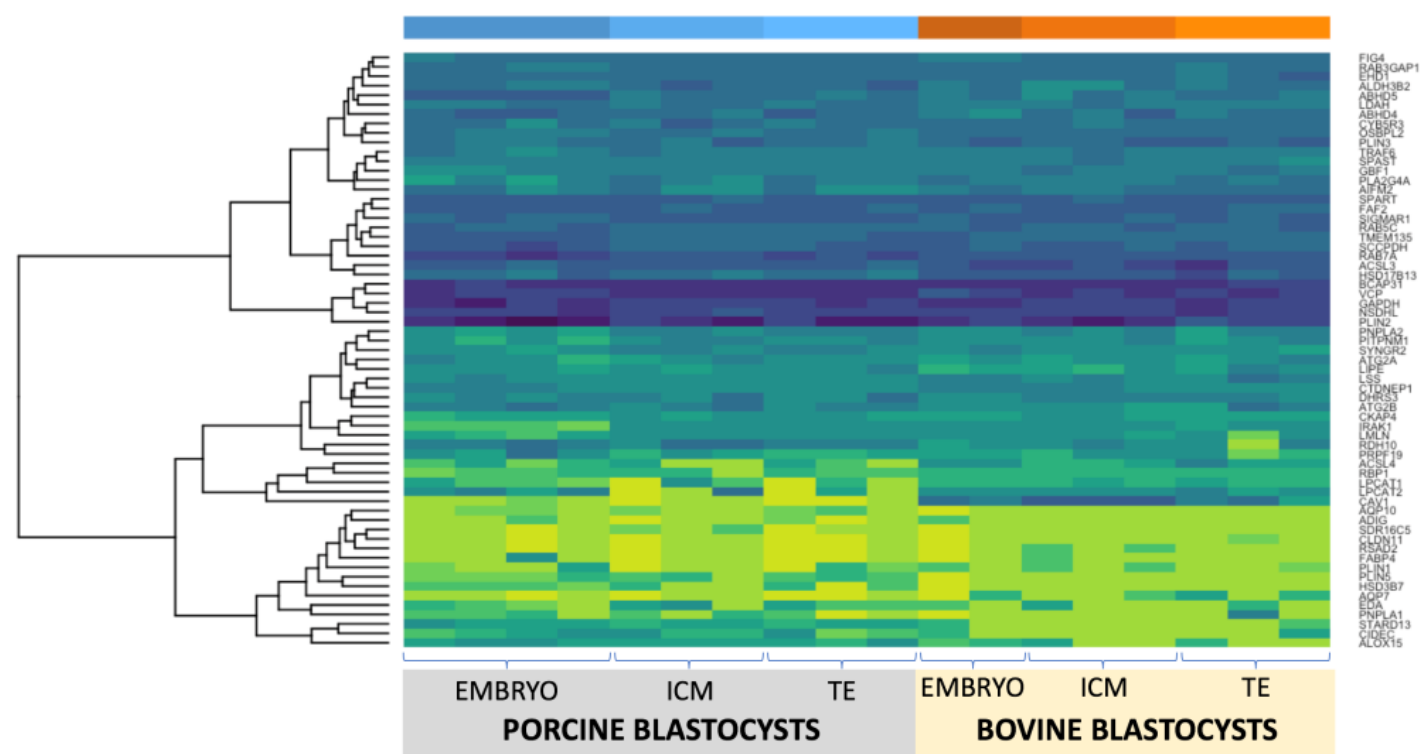

Figure 3. Heatmap of gene ontology (GO) term "lipid droplet" representing porcine and bovine parthenogenetic, blastocyst stage embryos, inner cell mass (ICM) and trophoblast (TE) cell lines. 


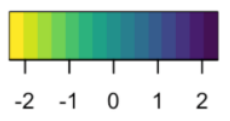

Column Z-Score

\section{Lipid storage GO term}

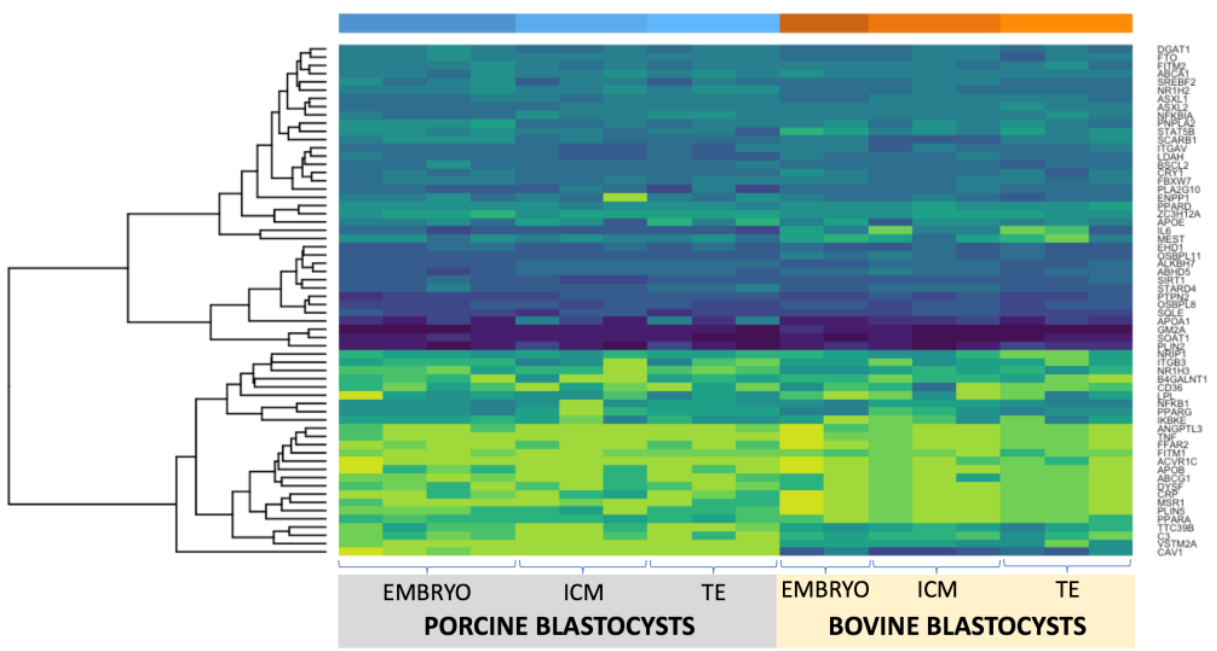

Figure 4. Heatmap of gene ontology (GO) term "lipid storage" representing porcine and bovine parthenogenetic, blastocyst stage embryos, ICM and TE cell lines.

In whole embryos, GM2A, PLIN2, APOA1, SOAT1, SQLE, PTPN2, SIRT1, ALKBH7, OSBPL8, $A B H D 5$, STEARD4 were the most abundantly expressed genes in both species (APOA1, SQLE, SIRT1, OSBPL8 were not differentially expressed). PLIN2, GM2A and SOAT1 expression was outreach among other DEGs (Figures 5 and 6). In addition to these genes, we found CD36, SCARB1 and CAV1 abundantly expressed in the bovine ICM samples. TE cells abundantly expressed IL6 gene, which was upregulated in porcine samples.
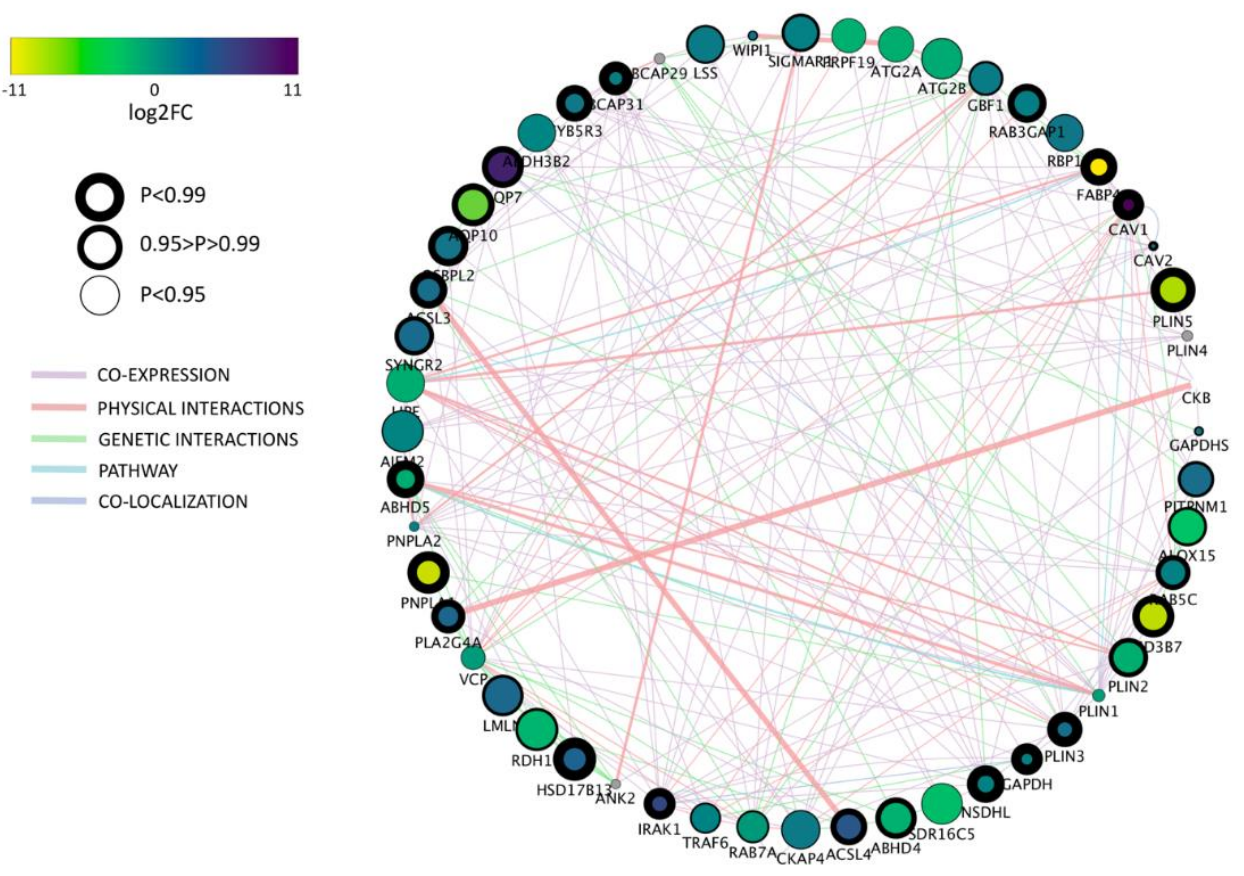

Figure 5. Network of differentially expressed genes (DEG) for GO term "lipid droplet" between porcine and bovine parthenogenetic, blastocyst stage embryos. Negative log2FC values indicate an upregulation of genes in porcine embryos. Node envelope thickness expressed as NOIseq probability value. 


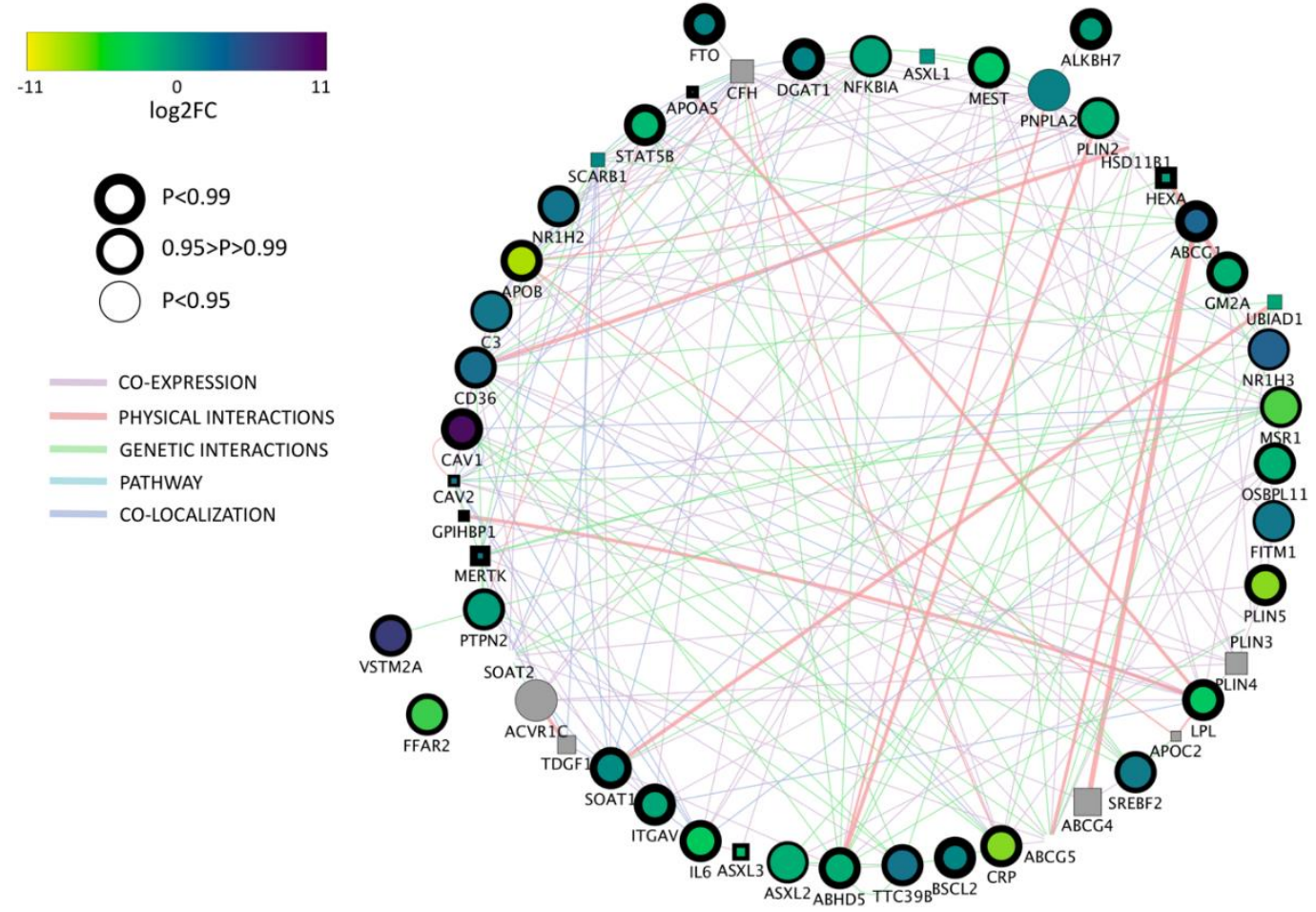

Figure 6. Network of differentially expressed genes (DEG) for GO term "lipid storage" between porcine and bovine parthenogenetic, blastocyst stage embryos. Negative log2FC values indicate an upregulation of genes in porcine embryos. Node envelope thickness expressed as NOIseq probability value.

\section{Discussion}

Lipids play a fundamental role in development of preimplantation embryos by providing energy and other factors, such as hormone precursors. Staining of lipid droplets revealed that both the number of LD per single blastomere and the LD volume were significantly higher in porcine blastocysts, in comparison to bovine. This observation may be supported by the distribution of LD in oocytes of both species, since significantly higher lipid content in porcine oocytes was noticed when compared to, e.g., bovine, human or murine counterparts [6]. Yet, the functional and biological relevance of high lipid stores in porcine oocytes and embryos still remains to be solved. In our research, we have used a model of parthenogenetic embryo development, which is routinely used in the in vitro porcine embryo production, and also serves as a source for the derivation of embryonic stem cell lines.

Based on the gene ontology (GO) term selection on "lipid droplet" we found 69.5\% (44 among 63) differentially expressed genes between bovine and porcine parthenogenetic blastocysts. Among them, PLIN2 and PLIN3 were found which play crucial roles in LD metabolism. PLIN2 prevents lipid degradation and hence promotes lipid accumulation, whereas PLIN3 can be recruited to the newly synthesized LD, suggesting its involvement in protection and trafficking of the nascent LD [11,19]. The results of our experiment showed higher expression of the PLIN2 gene in porcine blastocysts and, on the contrary, an upregulation of PLIN3 in bovine blastocysts. Since blastomeres of porcine blastocysts already exhibit high accumulation of lipids (as shown by fluorescent staining), recognizing high level of PLIN2 as a marker of cellular lipid accumulation is justified. Bovine blastocysts, on the other hand, were characterized by lower lipid accumulation. Thus, it may be anticipated that a prior activation of mechanisms, which increase the accumulation of energy sources may be required. This process may be controlled by the product of the PLIN3 gene associated with the newly formed LD. This observation may be supported by higher number of LD noted in bovine blastocysts calculated per whole embryo. The probable higher dynamics of LD formation in the bovine blastocysts may also be supported by higher expression of the NSDHL gene. Its product is involved in synthesis of cholesterol, which after 
esterification is one of the main components of the LD core [20]. Moreover, we have observed higher expression of the ACSL3 gene in bovine blastocysts, which is involved in the generation of long-chain acyl-CoA and modulates cellular fatty acid uptake [17]. Paczkowski et al. suggested that upregulation of ACSL3 may be an indicator of fatty acid oxidation (FAO) rise [21]. Therefore, the data described above indicates, that although bovine blastocysts contain less LD, their formation and FAO is more dynamic at the blastocyst stage when compared to porcine embryos.

One of the most intriguing finding concerns caveolin 1 (CAV1) expression in porcine and bovine blastocysts. Caveolin-deficient mouse adipocytes show disturbed LD formation, different protein and lipid composition, as well as a different size distribution of LD. It is suggested that caveolins seem to play an important role in LD maturation and biogenesis [22,23]. Knockout mice of CAV1 gene showed reduced whole body FAO [24]. Additionally, other experiments indicated that the loss of CAV1 (lack of expression) causes a switch from lipid toward glucose metabolism $[25,26]$. Since we have observed a very low level of caveolin 1 transcript in porcine blastocysts, it might suggest that these embryos may trigger the energy metabolism preferably towards glucose consumption. This assumption may be additionally corroborated by the upregulation of genes engaged in glucose metabolism observed in porcine blastocysts. We show significantly higher expression of glucose transporter 2 (GLUT2), as well as hexokinase 2 (HEX2) or PDHA1 genes in porcine vs. bovine blastocysts. These genes are important for transport and conversion of glucose and their higher expression indicates the increased utilization of glucose as an energy source. Unlike pig embryos, bovine parthenogenetic blastocysts expressed a high level of $C A V 1$ transcript, confirming their constant dependency on fatty acids as an energy source. However, low mRNA level of genes controlling glucose metabolism (HEX2, GLUT2) may indicate limited consumption of glucose by embryos of this species. From our data, it may be concluded that bovine parthenogenetic blastocysts set their energy metabolism rather on fatty acids, whereas porcine parthenogenetic blastocysts switch into glucose consumption. Moreover, it was previously suggested that the energy state of the embryo does not have to be solely granted to either glucose or fatty acids, but it might function as a fine-tuned adaptive mechanism, reliable on multiple environmental and embryo originating factors.

Blastocyst formation is characterized by differentiation of blastomeres into pluripotent inner cell mass (ICM) that will give rise to the embryo proper and trophectoderm (TE) differentiating to the extraembryonic tissues. The two cell lines express different lineage specific markers, however, little is known about the differences related to the functional metabolic pathways. Here, we report that porcine ICM and TE cells do not show differences in gene expression with respect to "lipid storage", and only one gene with very low expression level in "lipid droplet" GO terms. On the contrary, we found significant differences in gene expression between bovine ICM and TE with eight genes related to "lipid droplets" GO term and 23 for "lipid storage". In porcine blastocysts, only the PLIN1 gene was significantly different, showing higher expression in TE cells however, the expression was very low in both cell lines. PLIN2 was one of the most abundantly expressed gene in ICM and TE of both species. Although the expression was comparable in ICMs, the TE cells upregulated PLIN2 in porcine embryos, compared to bovine, which correspond to the overall LD number. Morphologically, porcine embryos have a lower number of blastomeres than bovine, and the ICM may be considered "non-obvious" due to strong accumulation of LD in embryo compartments. Therefore, more relevant differences may be found at later stages of development of porcine embryos, while in cattle, the ICM is already clearly established and easy to distinguish. Although the expression was low in bovine and porcine embryos, we noticed the presence of PLIN5 transcripts and upregulation in ICM and TE cells in pig, which may indicate its role in LD biogenesis in preimplantation embryos.

Considering the results obtained from fluorescence staining, we also analyzed "lipid storage" gene ontology term, which shares with GO "lipid droplet" only seven of 125 genes. We found the highest expression of GM2A (GM2 ganglioside activator) gene, significantly upregulated in porcine embryos. GM2 Activator, also known as SAP3 (Shingolipid Activator Protein 3), coded by the GM2A gene, is one of the components of the B-hexosaminidaseA (HEXA) complex necessary for the enzymatic 
processing of gangliosides, which are sialic acid-containing acidic glycosphingolipids. Gangliosides found in animal plasma membranes exhibit cell-type specificity and are implicated in various cellular functions, differentiation, signal transduction and immunity $[27,28]$. The HEXA gene in our study was also significantly higher expressed in porcine blastocysts which corresponds to upregulation of a substrate specific cofactor GM2A. Most of publications describe GM1, GM3 and GD1a gangliosides as important factors involved in the EGFR (epidermal growth factor receptor) signaling pathway [28]. In porcine embryos, exogenous GD1a supported maturation and blastocyst formation in vitro [29]. Others report GM1 and GM3 expression in theca and cumulus rat cells, while Ju et al. correlated GM3 expression with apoptosis in preimplantation mouse embryos [30]. To our knowledge, this is the first report on abundant expression of $G M 2 a$ in porcine and bovine preimplantation embryos. Due to known antioxidative, signal transduction and cell differentiation functions of gangliosides, the upregulation of GM2a may indicate relevant function during the time of ICM and TE formation. According to BioGRID, a genetic interaction (GeneMania) exist between HEXA and ABHD5 (also known as CGI-58), which is responsible for activation of PNPLA2 lipase after dissociation from phosphorylated perilipin on LD surface. Although ABHD5 was upregulated in porcine blastocyst, PNPLA2 lipase displayed similar expression level. Interestingly, LD associated hydrolase ( $L D A H)$ expression was increased in porcine ICM and TE cell lines, compared to bovine. It has been recently shown that $L D A H$ promote TAG accumulation and fusion of lipid droplets facilitating the metabolism towards lipid storage [31]. These results support the conclusion of Goo et al. that $L D A H$ plays primarily lipogenic role together with the inhibition of PNPLA2. The second the most abundantly expressed gene was SOAT1 (Sterol O-Acyltransferase 1). In mouse, microarray data showed that SOAT1 was one of the genes which expression significantly increased during morula to blastocyst transition, what may be related to the differentiation of the first two embryonic cell lineages [32]. The Soats genes contribute to cholesterol metabolism mainly by esterification to enable efficient uptake and transportation. It is believed that SOAT1 plays major role in maintenance of cholesterol homeostasis in cells and contribute to the accumulation of LD which is in line with our observations [33]. In our study, abundant SOAT1 expression was probably reflected with high expression of APOA1, a component of high-density lipoprotein (HDL) that promotes cholesterol efflux. Taking these results and the already mentioned upregulation of the NSDHL gene in bovine blastocyst together, we suggest a significant role of cholesterol metabolism during preimplantation development.

In conclusion, we have described significant differences in lipid metabolism between bovine and porcine parthenogenetic blastocysts. Aside from differences in lipid stores reflected by the LD number and volume, the parthenotes of both species also regulate lipid metabolism differentially on the gene expression level. Some genes, like CAV1 or GMA2, may be considered species specific and stage specific markers of the embryo metabolism. Other, such as FAB4, DGAT1, CD36 or NR1H3, although not highly expressed, show significant different between porcine and bovine embryos. This observation may therefore indicate lipid associated markers pointing to distinct regulatory mechanisms, which include cytoplasmic and nuclear factors. Genes characterized by the highest expression in both species, e.g., GMA2, PLIN2, APOA1, SOAT, GAPDH or BCAP31, may suggest their superior role in the regulation of metabolic pathways during blastocyst formation and cell differentiation.

\section{Materials and Methods}

All procedures were performed in accordance with the "Act on the protection of animals used for scientific purpose" of the Republic of Poland, which complies with the European Union Legislation for the protection of animals used for scientific purposes (Dz.U. 2015 poz. 266; 15 January 2015). According to these regulations ethics approval was not required, as the biological material (ovaries) was collected upon animal slaughter in abattoir (porcine-Sokolow S.A. Robakowo; bovine-Biernacki Sp. z o.o., Golina). Unless stated otherwise, all chemicals were purchased from Sigma. 


\subsection{Collection of Cumulus-Oocyte Complexes}

The cumulus-oocyte complexes (COCs) were collected post-mortem from bovine and porcine ovarian follicles of 3-6 mm diameter. Ovaries were transported in thermoisolated flask $2-4 \mathrm{~h}$ after slaughter to the laboratory. Ovaries were collected from pubertal gilts and heifers. Immature COCs were aspirated from ovarian follicles with needle and syringe and scored morphologically in HEPES-Talp medium. Only COCs presenting evenly granulated cytoplasm and at least 3-4 layers of cumulus cells were used in experiment.

\subsection{In Vitro Maturation}

In vitro maturation of porcine COCs was performed in $500 \mu \mathrm{L}$ of IVM medium (NCSU-23; North Carolina State University Medium-23) in four-well plates (Nunc, NY, USA). Each $500 \mu \mathrm{L}$ IVM medium consisted of 70 COCs that were matured in HeraCell 150 incubator (Thermo Scientific, MA, USA) under conditions: $5 \% \mathrm{CO}_{2}$ in atmosphere, $38.5^{\circ} \mathrm{C}$ and maximum humidity. The first $24 \mathrm{~h}$ of porcine IVM included medium supplemented with hormones: 10 U PMSG (pregnant mare serum gonadotropin, Chorulon, MSD Animal Health, NL, USA) and 10 U hCG (Folligon, MSD Animal Health, NL, USA). The next step included the transfer of COCs to fresh, equilibrated medium without hormones for $20 \mathrm{~h}$ maturation. The same batch of follicular fluid $(10 \% v / v)$ was used throughout the whole experiment. Bovine COCs were matured at $39{ }^{\circ} \mathrm{C}, 5 \% \mathrm{CO}_{2}$ at maximum humidity in TCM199 medium supplemented with $0.006 \mathrm{~g} / \mathrm{mL}$ fafBSA, $0.25 \mathrm{mM}$ Na-pyruvate, $1 \times$ conc. penicillin-streptomycin, $0.02 \mathrm{U} / \mathrm{mL} \mathrm{LH}, 0.002 \mathrm{mg} / \mathrm{mL}$ FHS and $0.001 \mathrm{mg} / \mathrm{mL} \beta$-estradiol for $24 \mathrm{~h}$. After IVM, COCs were denuded and all oocytes with first polar body, and no signs of degeneration were activated for parthenogenetic development.

\subsection{Embryo Culture}

Denuded oocytes were activated using $5 \mu \mathrm{M}$ ionomycin in TALP-medium ( $5 \mathrm{~min}$ ) and incubated in $2 \mathrm{mM}$ 6-DMAP for $4 \mathrm{~h}$ in final embryo culture medium supplemented with BSA (for the prevention of the second polar body extrusion). NCSU23 and SOF were used as embryo culture media up to blastocyst stage (7th day post activation for porcine embryos and 9th day for bovine). At day 5 of porcine embryo development, a half of the medium was changed with fresh medium, equilibrated and supplemented with $20 \%$ of FBS. At the 3rd day of bovine embryo development half of the medium was changed with fresh and equilibrated SOF. The blastocyst rate was calculated in relation to activated oocytes.

\subsection{Lipid Droplets Staining}

Porcine and bovine blastocysts were fixed in $4 \%$ PFA for $30 \mathrm{~min}$ at $37^{\circ} \mathrm{C}$ in four-well plates (Nunc). PFA was removed by washing the embryos twice in PBS with $0.2 \%$ PVP and stored at $4{ }^{\circ} \mathrm{C}$ for no longer than two weeks. Embryo permeabilization was performed with $0.2 \%$ Triton X-100 solution for $30 \mathrm{~min}$ at room temp. (RT) and washed $2 \times$ afterwards in $0.2 \%$ PVP/PBS. Lipid droplets were stained with fluorescent dye-20 $\mu \mathrm{g} / \mathrm{mL}$ BODIPY 493/503 (Thermo Scientific, Carlsbad, CA, USA) at room temperature for one hour. The chromatin of blastomeres were visualized by staining with $0.5 \mu \mathrm{g} / \mathrm{mL}$ DAPI (4',6-diamidino-2-phenylindole; Vector Laboratories, Burlingame, CA, USA). Embryos were mounted on glass slide with single concave (Comex, PL), coverslipped and analyzed using confocal microscope Zeiss LSM 880 using $488 \mathrm{~nm}$ filter with band pass 500-550 nm for BODIPY 493/503 (Laser Argon2) and 420-480 nm for DAPI (Laser Diode 405). LD were assessed through several optical sections (Z-stack) captured every $5 \mu \mathrm{m}$ to exclude double positioning of the same structures on two stacks. Objective (LD LCl Plan Apochromat 40×/1.2 Imm Korr DIC 27; Zeiss, Germany), pinhole, filters, offset settings were kept constant throughout the experiments. LD counting using ImageJ software (NIH, Bethesda, MD, USA) was previously described. 


\subsection{Sample Collection for RNA-seq}

Single porcine and bovine blastocyst stage embryos were selected from culture media and washed $3 \times$ in prewarmed PBS (ThermoFisher Scientific). SMART-Seq 44 Ultra Low Input RNA Kit for Sequencing (Clontech Takara) was used to generate high-quality cDNA from samples. The kit was designed to work with ultra-low amounts of total RNA (also at single cell level) and full-length transcripts. Samples were prepared according to manufacturer protocol. Firstly, $10 \times$ reaction buffer was prepared by combining $10 \times$ lysis solution and RNAse inhibitor. Embryos were transferred to $3 \mu \mathrm{L}$ of ultrapure water (Ambion, Austin, TX, USA) in 96 LoBind, semi-skirted Eppendorf twin.tec ${ }^{\circledR}$ PCR clean plate in the volume of $6.5 \mu \mathrm{L}$ PBS. Immediately $1 \mu \mathrm{L}$ of freshly made reaction buffer was added to embryos, vortexed gently and incubated at room temp. for $5 \mathrm{~min}$. Next, $3^{\prime}$ SMART-Seq CDS Primer II $(2 \mu \mathrm{L})$ was added to each sample, covered with foil and incubated for $3 \mathrm{~min}$. in $72{ }^{\circ} \mathrm{C}$ using Biometra thermocycler (TProfessional). Next a reaction mixture for first strand cDNA synthesis was added to each sample in the volume of $7.5 \mu \mathrm{L}$ and consisting of: $2 \mu \mathrm{L}$ SMARTScribe Reverse Transcriptase, $1 \mu \mathrm{L}$ of SMART-Seq v4 Oligonucleotide $(48 \mu \mathrm{M}), 4 \mu \mathrm{L}$ of $5 \times$ Ultra Low First-Strand Buffer, $0.5 \mu \mathrm{L}$ of RNase Inhibitor $(40 \mathrm{U} / \mu \mathrm{L})$. The reaction steps included $42{ }^{\circ} \mathrm{C}$ incubation for $90 \mathrm{~min}$. and $70{ }^{\circ} \mathrm{C}$ incubation for $10 \mathrm{~min}$. followed by transfer of plate on ice. Directly after cDNA synthesis it was amplified by LD PCR. A mixture of $25 \mu \mathrm{L} 2 \times$ SeqAmp PCR Buffer, $1 \mu \mathrm{L}$ PCR Primer II A $(12 \mu \mathrm{M})$, $1 \mu \mathrm{L}$ SeqAmp DNA Polymerase and $3 \mu \mathrm{L}$ Nuclease-Free water was added to each cDNA sample. The shaken and spinned plate was placed back into the Biometra thermocycler with the following protocol setup: initial denaturation: $95^{\circ} \mathrm{C} / 1 \mathrm{~min}$., amplification $\left(17\right.$ cycles): $98^{\circ} \mathrm{C} / 10 \mathrm{~s}, 65^{\circ} \mathrm{C} / 30 \mathrm{~s}$, $68^{\circ} \mathrm{C} / 3 \mathrm{~min}$., final extension in $72^{\circ} \mathrm{C}$ for $10 \mathrm{~min}$., followed by $4{ }^{\circ} \mathrm{C}$ storage until purification. cDNA was further cleaned and eluted prior quality check and library preparation. According to Clontech protocol a $50 \mu \mathrm{L}$ Agencourt AMPure XP beads and $1 \mu \mathrm{L}$ of lysis buffer were used at first to bind cDNA in final reaction volume for $10 \mathrm{~min}$ at room temperature. The 96-plate was placed on a magnetic stand (Ambion, Life Technologies) for 10 min., and the remaining solution was discarded. A freshly made $80 \%$ pure ethanol solution was applied to each sample well twice to wash the cDNA. Washing steps lasted for $2 \mathrm{~min}$, with the plate remaining on the magnetic stand. Finally, samples were air dried for 2-3 min. and re-suspended in $17 \mu \mathrm{L}$ of Elution Buffer. Samples were pipetted few times in order to ensure complete elution and dissociation from magnetic beads. The plate was placed on a magnetics stand for $5 \mathrm{~min}$., and the eluent was transferred to a fresh Low Bind $0.5 \mathrm{ml}$ Eppendorf tube.

\subsection{Library Preparation}

Double stranded cDNA was prepared from total RNA isolated from each sample with the use of SMARTer Ultra Low RNA Kit for Illumina Sequencing (Clontech Takara), according to user manual with 17 cycles of amplification. Final libraries were prepared from cDNA with the use of the Nextera XT DNA library preparation kit and Nextera XT Index Kit v2 (Illumina, San Diego, CA, USA). Quality and quantity of cDNA for each sample were assessed with Agilent Bioanalyzer and Qubit fluorometer. Library preparation was performed according to manufacturer instruction for single-cell workflow with 500 pg cDNA input (according to Qubit measurements) and 12 cycles of amplification. The size of final library constructs was assessed by means of on-chip electrophoresis with the use of Agilent Bioanalyzer and Agilent High Sensitivity DNA Kit. The concentration of libraries was measured by the use of a Qubit 4 fluorometer and QUBIT dsDNA HS assay (ThermoFisher Scientific). All final libraries were diluted with $10 \mathrm{mM}$ Tris-Cl to $2 \mathrm{nM}$, and then pooled by volume. Libraries were validated by shallow sequencing on Illumina MiSeq ${ }^{\circledR}$ System with the use of MiSeq ${ }^{\circledR}$ Reagent Kits v2 (500 cycles). A validation run was performed in $2 \times 250 \mathrm{bp}$ PE mode with $10 \mathrm{pM}$ libraries and 5\%PhiX spiked-in. The library pool was sequenced with $2 \times 150$ PE mode (NovaSeq ${ }^{\mathrm{TM}} 6000$, Illumina, San Diego, CA, USA). 


\section{7. mRNA Gene Expression}

qPCR was done using the standard curve method. For this purpose, the desired sequences for analyzed genes were amplified by PCR and visualized on 1.5\% agarose gel with the Gene Ruler ${ }^{\mathrm{TM}}$ $100 \mathrm{bp}$ DNA Ladder (Fermentas, Burlington, ON, Canada). The PCR product was extracted from the gel, isolated and purified using the Gel Extraction Kit (Fermentas, Burlington, ON, Canada). Based on the DNA concentration measured with a Nanodrop c2000 system (Thermo Scientific, Carlsbad, CA, USA), a serial 10-fold dilutions of a DNA with a known concentration (standards) were generated. Each standard was used as a separate template for a real-time PCR reaction to produce the appropriate standard curve with the LightCycler 480 II software (Roche, Basel, Switzerland). The reaction conditions and the efficiency of the reactions for all genes were analyzed separately. All reactions were performed using the Light Cycler $480 \mathrm{II}$ system with a set of supplied reagents. The $10 \mu \mathrm{L}$ reaction mixture consisted of $5 \mu \mathrm{L}$ of the LightCycler Probe Master, $0.5 \mu \mathrm{M}$ primers, $0.3 \mu \mathrm{M}$ probes and $1 \mu \mathrm{L}$ each of the cDNA. The reaction conditions were as follows: denaturation: $95^{\circ} \mathrm{C}, 10 \mathrm{~min}$; amplification: 40 cycles of $95{ }^{\circ} \mathrm{C}$ for $10 \mathrm{~s}, 60^{\circ} \mathrm{C}$ for $10 \mathrm{~s}, 72{ }^{\circ} \mathrm{C}$ for $10 \mathrm{~s}$; and final cooling at $40{ }^{\circ} \mathrm{C}$. The temperature slope was set at $20^{\circ} \mathrm{C} / \mathrm{s}$ during amplification.

\subsection{Bioinformatic Tools, Data Processing and Statistics}

Raw reads of Sus scrofa and Bos taurus were aligned to Ensembl Sscrofa11.1 and ARS-UCD1.2 genomes, respectively, with STAR (version 2.7). Reads mapped to exons were counted with featureCounts (version 1.6). List of orthologous genes in pigs and cows were obtained from the Ensembl database. For further analysis, we included orthologue genes with one-to-one matching and excluded duplicates, unmatched, one-to-many matching genes (16,384 genes left). Orthologue genes with no expression were removed from the analysis (15,023 genes remaining). Row reads were normalized with SCnorm package (version 1.8). Differential genes expression was calculated with NOISeq package (version 2.30). Heatmaps were created with M3Drop (version 1.12) and/or gplots (version 3.0) with basic plot functions of R. All R steps were performed in R version 3.6. Lists containing genes belonging to "lipid droplet" (GO:0005811), and "lipid storage" (GO:0019915) gene ontology terms were downloaded from the AmiGO 2 database. The selected, differentially expressed genes between porcine and bovine blastocysts of both GO terms have been uploaded to DAVID Bioinformatics Resources 6.3 and analyzed using a functional annotation tool. The minimum number of genes for the corresponding term was set as 3. EASE Score refers to the one-tail Fisher exact probability value, and was set as 0.05 . A comparison of LD characteristics between experimental groups was performed using IBM SPSS Statistics 23.0. All data (before computing) were subjected to testing for normal distribution using the Kolmogorov-Smirnov and Shapiro-Wilk tests. The differences in lipid droplet number and occupied volume in embryos were calculated using the Kruskal-Wallis and two-tailed Mann-Whitney U tests. Pearson correlation coefficient was calculated for lipid droplet number and volume association. All data with $p<0.05$ were considered statistically significant.

Supplementary Materials: The following are available online at http://www.mdpi.com/1422-0067/21/18/6488/s1. Author Contributions: Conceptualization-P.P.; methodology-P.P., E.W., A.K., N.D., Z.E.M.; writing-original draft preparation, P.P.; writing-review and editing, E.W., D.L. and J.W.; visualization-P.P., A.K.; supervision-P.P., J.W.; funding acquisition-P.P. All authors have read and agreed to the published version of the manuscript.

Funding: This research was funded by National Science Centre, Poland, grant number (2014/13/D/NZ2/03901).

Acknowledgments: We acknowledge veterinary doctor, Piotr Kempski for obtaining the tissues for research and Joanna Lechtanska for support in basic laboratory procedures and organization.

Conflicts of Interest: The authors declare no conflict of interest. The funders had no role in the design of the study; in the collection, analyses, or interpretation of data; in the writing of the manuscript, or in the decision to publish the results. 


\section{Abbreviations}

$\begin{array}{ll}\text { LD } & \text { Lipid droplet } \\ \text { NCSU23 } & \text { North Carolina State University 23 Medium } \\ \text { SOF } & \text { Synthetic Oviduct Fluid Medium } \\ \text { ICM } & \text { Inner Cell Mass } \\ \text { DMAP } & \text { (Dimethylamino)purine } \\ \text { TE } & \text { Trophectoderm } \\ \text { DEG } & \text { Differentially expressed gene } \\ \text { DAPI } & 4^{\prime}, 6 \text {-diamidino-2-phenylindole } \\ \text { FAO } & \text { Fatty acid oxidation }\end{array}$

\section{References}

1. Tang, F.; Barbacioru, C.; Wang, Y.; Nordman, E.; Lee, C.; Xu, N.; Wang, X.; Bodeau, J.; Tuch, B.B.; Siddiqui, A.; et al. mRNA-Seq whole-transcriptome analysis of a single cell. Nat. Methods 2009, 6, 377-382. [CrossRef]

2. Yan, L.; Yang, M.; Guo, H.; Yang, L.; Wu, J.; Li, R.; Liu, P.; Lian, Y.; Zheng, X.; Yan, J.; et al. Single-cell RNA-Seq profiling of human preimplantation embryos and embryonic stem cells. Nat. Struct. Mol. Biol. 2013, 20, 1131-1139. [CrossRef]

3. Madeja, Z.E.; Pawlak, P.; Piliszek, A. Beyond the mouse: Non-rodent animal models for study of early mammalian development and biomedical research. Int. J. Dev. Biol. 2019, 63, 187-201. [CrossRef]

4. Romek, M.; Gajda, B.; Krzysztofowicz, E.; Kepczyński, M.; Smorag, Z. Lipid content in pig blastocysts cultured in the presence or absence of protein and vitamin E or phenazine ethosulfate. Folia Biol. 2011, 59, 45-52. [CrossRef]

5. Pawlak, P.; Warzych, E.; Cieślak, A.; Malyszka, N.; Maciejewska, E.; Madeja, Z.E.; Lechniak, D. The consequences of porcine IVM medium supplementation with follicular fluid become reflected in embryo quality, yield and gene expression patterns. Sci. Rep. 2018, 8, 15306. [CrossRef]

6. Bradley, J.; Swann, K. Mitochondria and lipid metabolism in mammalian oocytes and early embryos. Int. J. Dev. Biol. 2019, 63, 93-103. [CrossRef] [PubMed]

7. McEvoy, T.G.; Coull, G.D.; Broadbent, P.J.; Hutchinson, J.S.M.; Speake, B.K. Fatty acid composition of lipids in immature cattle, pig and sheep oocytes with intact zona pellucida. J. Reprod. Fertil. 2000, 118, 163-170. [CrossRef] [PubMed]

8. Kikuchi, K.; Ekwall, H.; Tienthai, P.; Kawai, Y.; Noguchi, J.; Kaneko, H.; Rodriguez-Martínez, H. Morphological features of lipid droplet transition during porcine oocyte fertilisation and early embryonic development to blastocyst in vivo and in vitro. Zygote 2002, 10, 355-366. [CrossRef] [PubMed]

9. Walther, T.C.; Farese, R.V. Lipid droplets and cellular lipid metabolism. Annu. Rev. Biochem. 2012, 81, 687-714. [CrossRef]

10. Welte, M.A. Expanding roles for lipid droplets. Curr. Biol. 2015, 25, R470-R481. [CrossRef]

11. Bickel, P.E.; Tansey, J.T.; Welte, M.A. PAT proteins, an ancient family of lipid droplet proteins that regulate cellular lipid stores. Biochim. Biophys. Acta Mol. Cell Biol. Lipids 2009, 1791, 419-440. [CrossRef] [PubMed]

12. Tauchi-Sato, K.; Ozeki, S.; Houjou, T.; Taguchi, R.; Fujimoto, T. The Surface of Lipid Droplets Is a Phospholipid Monolayer with a Unique Fatty Acid Composition. J. Biol. Chem. 2002, 277, 44507-44512. [CrossRef] [PubMed]

13. Dunning, K.R.; Russell, D.L.; Robker, R.L. Lipids and oocyte developmental competence: The role of fatty acids and $\beta$-oxidation. Reproduction 2014, 148, R15-R27. [CrossRef] [PubMed]

14. Watanabe, H.; Suzuki, H.; Fukui, Y. Fertilizability, developmental competence, and chromosomal integrity of oocytes microinjected with pre-treated spermatozoa in mice. Reproduction 2010, 139, 513-521. [CrossRef]

15. Bradley, J.; Pope, I.; Masia, F.; Sanusi, R.; Langbein, W.W.; Swann, K.; Borri, P. Quantitative imaging of lipids in live mouse oocytes and early embryos using CARS microscopy. Development 2016, 143, 2238-2247. [CrossRef]

16. Romek, M.; Gajda, B.; Krzysztofowicz, E.; Smorag, Z. Changes of lipid composition in non-cultured and cultured porcine embryos. Theriogenology 2010, 74, 265-276. [CrossRef]

17. Sudano, M.J.; Rascado, T.D.; Tata, A.; Belaz, K.R.; Santos, V.G.; Valente, R.S.; Mesquita, F.S.; Ferreira, C.R.; Araújo, J.P.; Eberlin, M.N.; et al. Lipidome signatures in early bovine embryo development. Theriogenology 2016, 86, 472-484.e1. [CrossRef] 
18. Sturmey, R.; Reis, A.; Leese, H.; McEvoy, T.; Sturmey, R.G. Role of Fatty Acids in Energy Provision During Oocyte Maturation and Early Embryo Development. Reprod. Domest. Anim. 2009, 44, 50-58. [CrossRef]

19. Skinner, J.R.; Shew, T.M.; Schwartz, D.M.; Tzekov, A.; Lepus, C.M.; Abumrad, N.A.; Wolins, N.E. Diacylglycerol enrichment of endoplasmic reticulum or lipid droplets recruits perilipin 3/TIP47 during lipid storage and mobilization. J. Biol. Chem. 2009, 284, 30941-30948. [CrossRef]

20. Shimobayashi, S.F.; Ohsaki, Y. Universal phase behaviors of intracellular lipid droplets. Proc. Natl. Acad. Sci. USA 2019, 116, 25440-25445. [CrossRef]

21. Paczkowski, M.; Schoolcraft, W.B.; Krisher, R.L. Fatty acid metabolism during maturation affects glucose uptake and is essential to oocyte competence. Reproduction 2014, 148, 429-439. [CrossRef] [PubMed]

22. Pol, A.; Luetterforst, R.; Lindsay, M.; Heino, S.; Ikonen, E.; Parton, R.G. A Caveolin Dominant Negative Mutant Associates with Lipid Bodies and Induces Intracellular Cholesterol Imbalance. J. Cell Biol. 2001, 152, 1057-1070. [CrossRef] [PubMed]

23. Blouin, C.; Le Lay, S.; Eberl, A.; Koefeler, H.; Guerrera, I.C.; Klein, C.; Le Liepvre, X.; Lasnier, F.; Bourron, O.; Gautier, J.-F.; et al. Lipid droplet analysis in caveolin-deficient adipocytes: Alterations in surface phospholipid composition and maturation defects. J. Lipid Res. 2009, 51, 945-956. [CrossRef] [PubMed]

24. Asterholm, I.W.; Mundy, D.I.; Weng, J.; Anderson, R.G.W.; Scherer, P.E. Altered Mitochondrial Function and Metabolic Inflexibility Associated with Loss of Caveolin-1. Cell Metab. 2012, 15, 171-185. [CrossRef] [PubMed]

25. Fernandez, M.A.; Restall, C.; Ferguson, C.; Martel, N.; Martin, S.; Bosch, M.; Kassan, A.; Leong, G.M.; Martin, S.D.; McGee, S.L.; et al. Caveolin-1 orchestrates the balance between glucose and lipid-dependent energy metabolism: Implications for liver regeneration. Hepatology 2012, 55, 1574-1584. [CrossRef]

26. Shiroto, T.; Romero, N.; Sugiyama, T.; Sartoretto, J.L.; Kalwa, H.; Yan, Z.; Shimokawa, H.; Michel, T. Caveolin-1 Is a Critical Determinant of Autophagy, Metabolic Switching, and Oxidative Stress in Vascular Endothelium. PLoS ONE 2014, 9, e87871. [CrossRef]

27. Mahuran, D.J. Biochemical consequences of mutations causing the GM2 gangliosidoses. Biochim. Biophys. Acta (BBA) Mol. Basis Dis. 1999, 1455, 105-138. [CrossRef]

28. Kim, B.H.; Ju, W.S.; Kim, J.-S.; Kim, S.-U.; Park, S.J.; Ward, S.M.; Lyu, J.H.; Choo, Y.-K. Effects of Gangliosides on Spermatozoa, Oocytes, and Preimplantation Embryos. Int. J. Mol. Sci. 2019, 21, 106. [CrossRef]

29. Kim, J.-W.; Park, H.-J.; Chae, S.-K.; Ahn, J.-H.; Do, G.-Y.; Choo, Y.-K.; Park, J.J.; Jung, B.D.; Kim, S.-U.; Chang, K.-T.; et al. Ganglioside GD1a promotes oocyte maturation, furthers preimplantation development, and increases blastocyst quality in pigs. J. Reprod. Dev. 2016, 62, 249-255. [CrossRef]

30. Ju, E.-J.; Kwak, D.-H.; Lee, D.-H.; Kim, S.-M.; Kim, J.-S.; Choi, H.-G.; Jung, K.-Y.; Lee, S.U.; Do, S.-I.; Park, Y.-I.; et al. Pathophysiological implication of ganglioside GM3 in early mouse embryonic development through apoptosis. Arch. Pharmacal Res. 2005, 28, 1057-1064. [CrossRef]

31. Goo, Y.-H.; Son, S.-H.; Paul, A. Lipid Droplet-Associated Hydrolase Promotes Lipid Droplet Fusion and Enhances ATGL Degradation and Triglyceride Accumulation. Sci. Rep. 2017, 7, 2743. [CrossRef] [PubMed]

32. Hamatani, T.; Carter, M.G.; Sharov, A.A.; Ko, M.S. Dynamics of Global Gene Expression Changes during Mouse Preimplantation Development. Dev. Cell 2004, 6, 117-131. [CrossRef]

33. Chang, N.-Y.; Chan, Y.-J.; Ding, S.-T.; Lee, Y.-H.; Huangfu, W.-C.; Liu, I.-H. Sterol O-Acyltransferase 2 Contributes to the Yolk Cholesterol Trafficking during Zebrafish Embryogenesis. PLoS ONE 2016, 11, e0167644. [CrossRef] [PubMed]

(C) 2020 by the authors. Licensee MDPI, Basel, Switzerland. This article is an open access article distributed under the terms and conditions of the Creative Commons Attribution (CC BY) license (http://creativecommons.org/licenses/by/4.0/). 